\title{
Testing models of speciation from genome sequences: divergence and asymmetric admixture in Island South- East Asian Sus species during the Plio-Pleistocene climatic fluctuations
}

\author{
LAURENT A. F. FRANTZ,${ }^{* 1}$ OLE MADSEN, ${ }^{*}$ HENDRIK-JAN MEGENS, ${ }^{*}$ MARTIEN A. M. \\ GROENEN* and KONRAD LOHSE $\uparrow^{1}$ \\ *Animal Breeding and Genomics Group, Wageningen University, De Elst 1, Wageningen, WD 6708, The Netherlands, \\ $\uparrow$ Institute of Evolutionary Biology, University of Edinburgh, Edinburgh EH93FL, UK
}

\begin{abstract}
In many temperate regions, ice ages promoted range contractions into refugia resulting in divergence (and potentially speciation), while warmer periods led to range expansions and hybridization. However, the impact these climatic oscillations had in many parts of the tropics remains elusive. Here, we investigate this issue using genome sequences of three pig (Sus) species, two of which are found on islands of the Sundashelf shallow seas in Island South-East Asia (ISEA). A previous study revealed signatures of interspecific admixture between these Sus species (Genome biology, 14, 2013, R107). However, the timing, directionality and extent of this admixture remain unknown. Here, we use a likelihood-based model comparison to more finely resolve this admixture history and test whether it was mediated by humans or occurred naturally. Our analyses suggest that interspecific admixture between Sunda-shelf species was most likely asymmetric and occurred long before the arrival of humans in the region. More precisely, we show that these species diverged during the late Pliocene but around $23 \%$ of their genomes have been affected by admixture during the later Pleistocene climatic transition. In addition, we show that our method provides a significant improvement over D-statistics which are uninformative about the direction of admixture.
\end{abstract}

Keywords: conservation genetics, genomics, hybridization, phylogeography, population genetics, speciation

Received 23 July 2014; revision received 1 October 2014; accepted 3 October 2014

\section{Introduction}

Over the last four million years, the Earth has undergone frequent climatic oscillations including many ice ages (Zachos et al. 2001; Miller et al. 2005). Genetic studies have revealed that these large scale climatic fluctuations played a critical role in the evolutionary history of contemporary species (Hewitt 2000, 2004). Recent studies making use of the increased power afforded by genome-scale data have allowed to test

Correspondence: Laurent A.F. Frantz, Fax: +31317482335;

E-mail: laurent.frantz@wur.nl

${ }^{1}$ Both authors equally contributed to this work. increasingly finer hypotheses regarding the existence and the timing of post-divergence gene flow (i.e. Lawniczak et al. 2010; Rohland et al. 2010; Cahill et al. 2013; Hearn et al. 2014).

The impact that quaternary climatic fluctuations had on speciation is highly dependent on the taxa and the geographic range (Stewart et al. 2010). In many temperate regions, range contractions into refugia during glacial periods likely promoted divergence (and speciation), while range expansions out of refugia during warm periods resulted in hybridization. However, we know a lot less about the Pleistocene history of less well-studied biodiversity hotspots in the tropics (Hewitt 2004, 2011). 
In this study, we investigate the history of divergence and admixture of three species of pigs (genus Sus) from Island South-East Asia (ISEA). The ISEA archipelago comprises thousands of islands on multiple tectonic plates (Hall 1998). While the islands of Borneo, Sumatra and Java and the Malay Peninsula form a large continental shelf known as the Sunda-shelf (Fig. 1), other Island clusters such as the Philippines are on different plates. Islands on the same continental shelf are often separated by shallow seas and, given the large scale climatic fluctuations during the Pliocene and Pleistocene and the resulting sea level changes, were connected by land bridges on many occasions (Hall 1998; Voris 2000). In particular, the sharp climatic transition in the midPleistocene (around 700KY) resulted in more frequent glacial cycles and hence exposure of the Sunda-Shelf (Elderfield et al. 2012). However the effect of this climatic transition on forest cover and the history of those species that depend on it, remains controversial (Gathorne-Hardy et al. 2002; Bird et al. 2005; Cannon et al. 2009; Wurster et al. 2010; Slik et al. 2011).
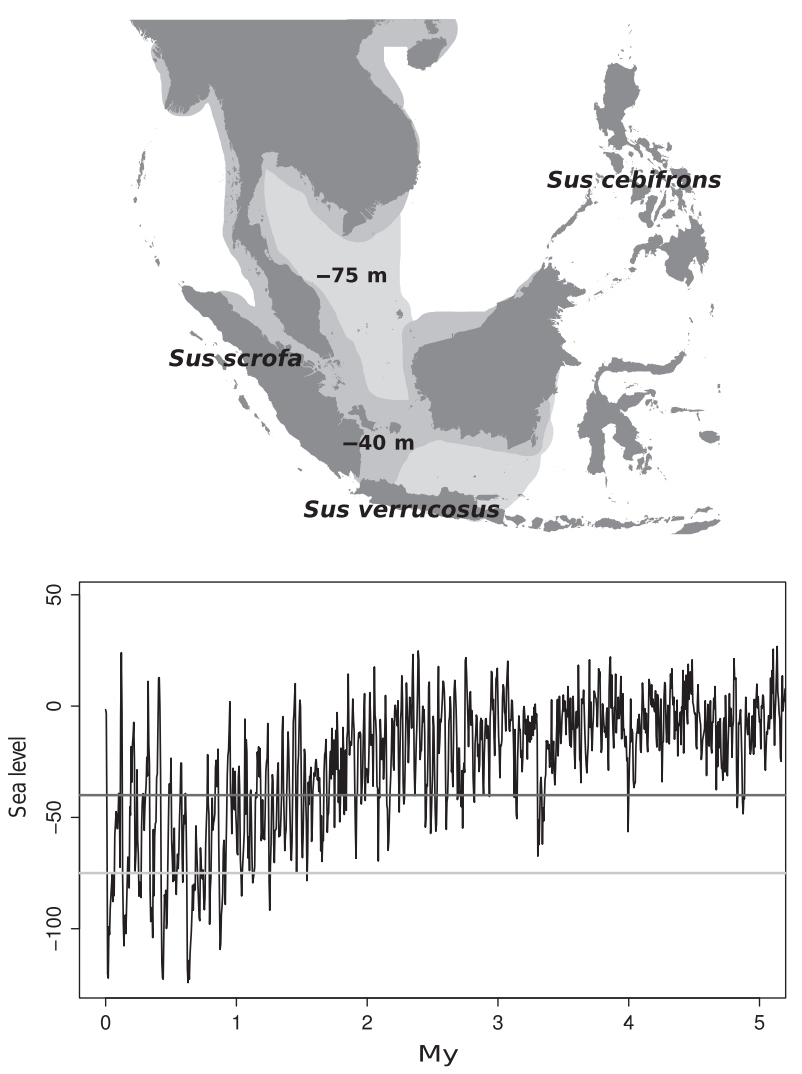

Fig. 1 Map of Island South-East Asia (upper part) with sea level fluctuations (lower part) over the last 4My (adapted from Miller et al. 2005). Dark grey and light grey areas on the map represent the exposure of the Sunda-shelf at $40 \mathrm{~m}$ and $75 \mathrm{~m}$ below current sea level, respectively.
The aim of this study is to characterize the speciation history of pig species in the genus Sus in ISEA. We focus on three species: Sus verrucosus (Java warty pig; Java, Indonesia), Sus cebifrons (Visayan warty pig; Philippines) and Sus scrofa (Eurasian wildboar; mainland Eurasia, Sumatra and North Africa). Most species in the genus Sus, such as $S$. verrucosus and S. cebifrons, are endemic to a single or few islands of ISEA (Meijaard et al. 2011). In contrast, S. scrofa is a widely distributed species with a natural range extending to most of Eurasia, North Africa and part of ISEA (Sumatra; Meijaard et al. 2011). In addition, this species has been introduced by humans into multiple regions of the world such as North America, Australia and Java (Meijaard et al. 2011). A previous study showed that $S$. verrucosus from Java (Sunda-shelf) is more closely related to $S$. cebifrons (Visayan Warty pig) in the Philippines than to S. scrofa (Eurasian wild boar) on Sumatra (Sunda-shelf; Fig. 1; Frantz et al. 2013). Moreover, this study showed that subsequent interspecific admixture likely took place on the Sunda-shelf, between S. scrofa and S. verrucosus after S. cebifrons diverged in the Philippines (Frantz et al. 2013). However, the timing, magnitude direction of admixture remains unknown. Firstly, it is unclear whether this interspecific admixture occurred naturally at all or, alternatively, whether it was the result of human-mediated translocation of pig species in ISEA (Groves 1984; Heinsohn 2003; Larson et al. 2005, 2007; Frantz et al. 2013) during the last $70 \mathrm{Ky}$ (Mijares et al. 2010). This is crucial for conservation efforts such as ex-situ breeding programmes, particularly for the endangered Java Warty pig S. verrucosus (Semiadi et al. 2008) and the critically endangered Visayan warty pig S. cebifrons (Oliver 2008). Secondly, if admixture was natural, we would like to understand its temporal context. For example, both divergence and hybridization could be the result of the mid-Pleistocene sharp climatic transition. Alternatively, these species may have diverged much earlier during the late Pliocene or early Pleistocene, when connections between islands on the Sunda-shelf were less frequent (Frantz et al. 2013; Fig. 1) and admixed again during the more frequent and intense glacial period of the latter Pleistocene.

In this study, we analysed three genomes of Sus from ISEA in a likelihood framework to i) determine whether interspecific admixture between $S$. verrucosus and $S$. scrofa is linked with recent human activities and ii) quantify the timing, extent and directionality of this admixture.

\section{Material and methods}

\section{Data set}

We used a genomic data set from three species of South-East Asian pigs that was previously analysed 
using phylogenetic methods and D-statistics (Frantz et al. 2013). The data set comprises a single unphased diploid genome sampled from a Eurasian wild boar Sus scrofa (Sumatran population; Fig. 1) and the two island endemics Sus verrucosus (Java; Fig. 1) and Sus cebifrons (Philippines; Fig. 1). Triplet alignments were rooted using Phacochoerus africanus (common African warthog) as an outgroup. These genomes were sequenced at $10-20 \times$ depth of coverage and aligned to the S. scrofa reference genome (Ssc10.2; Bosse et al. 2012; Groenen et al. 2012; Frantz et al. 2013). The likelihood method we use fits explicit models of species divergence and admixture from multilocus data (see likelihood method) and requires short blocks of phased sequence (within which recombination can be ignored) of equal length (Lohse et al. 2011; Hearn et al. 2014; Lohse \& Frantz 2014). We divided the reference genome of S. scrofa into 500 and $1000 \mathrm{bp}$ blocks. To ensure enough coverage to call all heterozygous sites in each block and to remove possible copy number variants (Paudel et al. 2013), we filtered out, for each species, any block that had an average read depth of coverage lower than $7 \times$ or higher than twice the genomewide average (Frantz et al. 2013) using the pileup format in SAMtools v0.1.12 ( $\mathrm{Li}$ et al. 2009). Clusters of two or more single nucleotide polymorphisms (SNPs) in a $10 \mathrm{bp}$ window were filtered out as well as SNPs within $3 \mathrm{bp}$ of an indel. We remo-ved blocks for which less than $90 \%$ of the sites were covered and excluded any site that had an effective coverage (Gronau et al. 2011) below 4. Lastly, we only selected blocks that passed the above filtering criteria in all four samples. We then randomly phased these diploid blocks as a previous study showed that maximum likelihood estimates (MLE; Lohse \& Frantz 2014) are robust to phasing error provided blocks are short. Although the data were phased at random, the low heterozygosity - only $0.12 \%$ of sites were heterozygous in the S. scrofa individual from Sumatra, the most out-bred sample (Bosse et al. 2012; Frantz et al. 2013) - meant that the majority $(67 \%)$ of $500 \mathrm{bp}$ sequences alignments contained at most one heterozygous site per individual and so were immune to phasing error. Violations of the 4-gamete criterion within a block can arise either due to recombination or back mutation, both of which are not compatible with the assumption of the model (Lohse \& Frantz 2014). We therefore excluded blocks containing more than one type of shared derived mutation $(6.6 \%$ and $15.6 \%$ in the 500-bp and $1 \mathrm{~kb}$ data sets, respectively). After applying these filtering steps to the entire pig autosome, we were left with 232373 and 190692 of 500-bp and 1-kbp blocks, respectively.
Models

We compared the fit of five nested models to test different scenarios for the evolutionary history of these species (Fig. 2). All our models assume the order of species divergence inferred by Frantz et al. 2013 as (S. scrofa, (S. cebifrons, S. verrucosus)) and have at least three parameters, the species divergence time $T_{1}$ (divergence of $S$. verrucosus and $S$. cebifrons), $T_{2}$ (the species divergence of $S$. scrofa and S. verrucosus/S. cebifrons) and a single $N_{e}$ parameter (constant effective population size). Based on D-statistics analysis (Green et al. 2010; Durand et al. 2011), we assumed that interspecific gene flow takes place between $S$. verrucosus and S. scrofa after the divergence of S. cebifrons (Frantz et al. 2013; Data S1, Supporting information). We first assessed the fit of the most complex/general history of instantaneous bidirectional admixture (IBA; Fig. 2e), that is a scenario in which admixture between $S$. scrofa and $S$. verrucosus is assumed to happen in both directions. This model involves two admixture parameters $\left(f_{1}\right.$ and $\left.f_{2}\right)$ and six parameters in total: $T_{1}, T_{2}, f_{1}, f_{2}, T_{g f}$ (time of admixture) and $N_{e}$. We then assessed the fit of different model simplifications: (i) a model of symmetrical admixture (ISA; Fig. $2 d, f_{1}=f_{2}=f$ ) and (ii) models of instantaneous unidirectional admixture (IUA) Fig. $2 b$ and c) in which admixture goes only one way (either S. scrofa $\rightarrow$ S. verrucosus [IUA_SS] or S. verrucosus $\rightarrow$ S. scrofa [IUA_SV]). These are special cases of the IBA model in which we set either $f_{1}=0$ or $f_{2}=0$ (Fig. 2) and so have five parameters. Lastly, we evaluated the support of a simple divergence model (DIV; Fig. 2a) with no interspecific admixture, that is $\mathrm{f}_{1}=f_{2}=0$.

The assumption of equal population size for all these species may be unrealistic. To test whether adding additional demographic parameters improved model fit, we also evaluated the support of the IUA models with different population size. We tested additional models in which we allowed either S. scrofa or both $S$. verrucosus and the ancestral population of $S$. verrucosus and $S$. cebifrons to have a different $N_{e}$ than the common ancestor of all three species (note that given the sampling scheme, we have no information about the $N_{e}$ of $S$. cebifrons). Because a model with two $N_{e}$ parameters and two admixture fractions is nonidentifiable with minimal samples, we only assessed the influence of extra $N_{e}$ parameters on the IUA models (IUA_SS and IUA_SV; Table S1, Supporting information).

\section{Likelihood analysis}

Polymorphism information in a block of sequences from three species can be summarized as a vector of counts of mutations on different genealogical branches. 
(a)
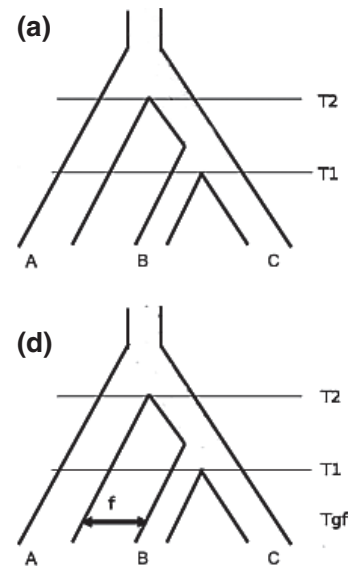

(b)
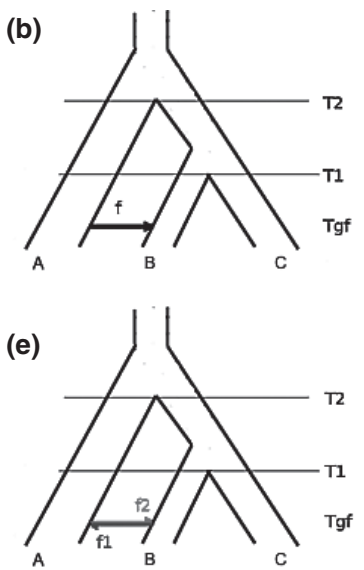

(c)

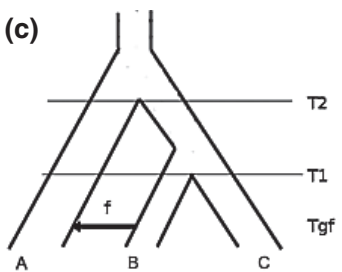

Fig. 2 Schematic representation of the five models tested in this study: a) Strict divergence (DIV without admixture or divergence with) b) with admixture from S. scrofa to S. verrucosus (IUA_SS), c) admixture from $S$. verrucosus to $S$. scrofa (IUA_SV), d) symmetrical admixture (ISA) and e) bidirectional admixture (IBA).

For a polarized sample (know ancestral state) of three sequences, there are six possible mutations types: three private and three shared mutations. We will hereafter refer to this vector as the mutational configuration of a block. Lohse et al. (2011, eqn 1) have shown that the probability of an observed mutational configuration in a particular block can be expressed as a higher order derivative of the generating function of genealogical branch lengths. The generating function for triplet samples for the IUA models is described in Hearn et al. (2014) and Lohse \& Frantz (2014). We give analogous results for the more general case of the IBA model in the supplementary information (Data S1, Supporting information). Assuming (initially) that blocks are unlinked (hence statistically independent observations), the logarithm of the likelihood (lnL) for a particular model is the sum of the $\ln L$ across blocks. We maximize the likelihood numerically using Mathematica v10. To correct for the effect of linkage when comparing models, we rescaled the difference in $\ln L$ between models as described in Lohse \& Frantz (2014). We assumed that the effect of physical linkage between blocks separated by a distance of $100 \mathrm{~kb}$ can be ignored (Tortereau et al. 2012). Further details of the general method for computing likelihoods and $95 \%$ CI of parameters are given in Lohse et al. (2011) and Lohse \& Frantz (2014). For each model, we computed
$\Delta \ln L$, the difference in $\log$ likelihood to the best fitting model. We assessed statistical support between nested models with a likelihood ratio test and assumed that $2 * \Delta \ln L$ follows a $\chi^{2}$ distribution with degree of freedom equal to the difference in the number of parameters of the two models (see Table 1).

To compare our approach with the D-statistics (Green et al. 2010; Durand et al. 2011) and to obtain a rough assessment of goodness of fit, we computed the expected counts of ABBA and BABA sites and E[D] from the generating function under the different admixture models (by fixing parameters to their MLE estimated from the data; see Data S1, Supporting information).

To scale relative time estimates into absolute values, we assumed an average divergence time between the African warthog and the ingroup of 10.5 MY and a generation time of 5 years (Gongora et al. 2011; Groenen et al. 2012; Frantz et al. 2013).

\section{Results}

\section{Model comparison}

By definition, the IBA model (Fig. 2e) provided a better fit than any of the simpler nested models (Table 1). Setting admixture fractions to be equal $\left(f_{1}=f_{2}=f\right.$;

Table 1 Model description and difference in support compared to best fitting model for $500 \mathrm{bp}$ and $1 \mathrm{~kb}$ blocks

\begin{tabular}{|c|c|c|c|}
\hline $\begin{array}{l}\text { Acronym } \\
\text { (parameters) }\end{array}$ & Description & $\Delta \operatorname{lnL}(500)$ & $\Delta \operatorname{lnL}(1 \mathrm{k})$ \\
\hline DIV (3) & Strict divergence (no gene flow) with or without ancestral substructure (Fig. 2a) & $-49.3 * *$ & $-80.1 * *$ \\
\hline IUA_SS (5) & Divergence with gene flow from S. scrofa to S. verrucosus (Fig. 2b) & $-22.8^{* *}$ & $-26.8 * *$ \\
\hline IUA_SV (5) & Divergence with gene flow from S. verrucosus to $S$. scrofa (Fig. 2c) & -1.7 N.S. & -1.6 N.S. \\
\hline ISA (5) & Symmetrical admixture model with equal admixture fractions (Fig. 2d) & $-10.1^{* *}$ & $-9.24 * *$ \\
\hline IBA (6) & Bidirectional admixture model with independent admixture fraction (Fig. 2e) & 0 & 0 \\
\hline
\end{tabular}

Significance was obtained using a likelihood ratio test $(2 * \Delta \ln L)$ and assuming a chi-square distribution $(* * P<0.001 ; * P<0.01)$ 
Fig. 2d) significantly reduced the fit (ISA model; $\Delta \ln L=-10.1$ and -9.24 for 500 and $1 \mathrm{~kb}$, respectively; Table 1). This difference in likelihood is highly significant assuming a $\chi^{2}$ distribution $(P<0.001$; Table 1$)$. Likewise, a model in which $f_{1}=0$ (IUA_SS; Fig. 2c), that is a history with admixture only from Sus scrofa into Sus verrucosus also gave a significantly worse fit $(\Delta \ln L=-22.8$ and -26.8 for 500 and $1 \mathrm{~kb}$, respectively; Table 1). In contrast, setting $f_{2}=0$ (IUA_SV model; Fig. $2 b$ ) only marginally reduced the fit $(\Delta \ln L=-1.77[P>0.05]$ and -1.68 [P>0.05] for 500 and $1 \mathrm{~kb}$, respectively; Table 1$)$. Thus, a model of unidirectional admixture from $S$. verrucosus into $S$. scrofa cannot be rejected.

Lastly, a strict divergence model, that is $f_{1}=f_{2}=0$ (DIV model; Fig. 2a) provided a significantly worse fit $(\Delta \operatorname{lnL}=-49.3$ and -80.1 for 500 and $1 \mathrm{~kb}$, respectively; Table 1). These results demonstrate that this genomic data set contains a strong signal of interspecific admixture between $S$. scrofa and $S$. verrucosus, but surprisingly most of this admixture occurred from $S$. verrucosus into S. scrofa, so in the opposite direction than that assumed by previous studies (Frantz et al. 2013).

Including additional $N_{e}$ parameter for different populations (see Methods) did not significantly improve the fit (Table S1, Supporting information). To get a sense of how well different admixture histories explain the data, we computed the expected D statistic (E[D]) under each admixture scenario and compared it to the observed value. This also allowed us to assess the sensitivity of $\mathrm{D}$ to admixture in different directions. Constraining admixture to be from S. scrofa to S. verrucosus (the best fitting IUA_SV model) gives E [D] $=0.22$, while limiting admixture to the opposite direction only (the IUA_SS model) gives $E[D]=0.12$. The observed $\mathrm{D}$ of 0.175 (for $0.5 \mathrm{~kb}$ data) is in between and matches E[D] under the estimated IBA model $\mathrm{E}[\mathrm{D}]=0.16$. Thus, the unidirectional models both fit the data worse than the bidirectional scenario (IBA). Comparing the number of mutations on external branches for each of the three possible topologies expected under the IBA model to the observed spectrum of mutation counts reveals a tight fit (Fig. 3), suggesting that the IBA model explains most of the signal in the data.

\section{Parameter inference}

Maximum-likelihood estimates for each parameter (Fig. 4) were obtained under the best fitting models (IBA and IUA_SV). Our first goal was to determine whether the admixture between S. scrofa and S. verrucosus could have been mediated by humans. The marginal curves for the time of admixture, under both IBA and IUA_SV models, show very little support for values of

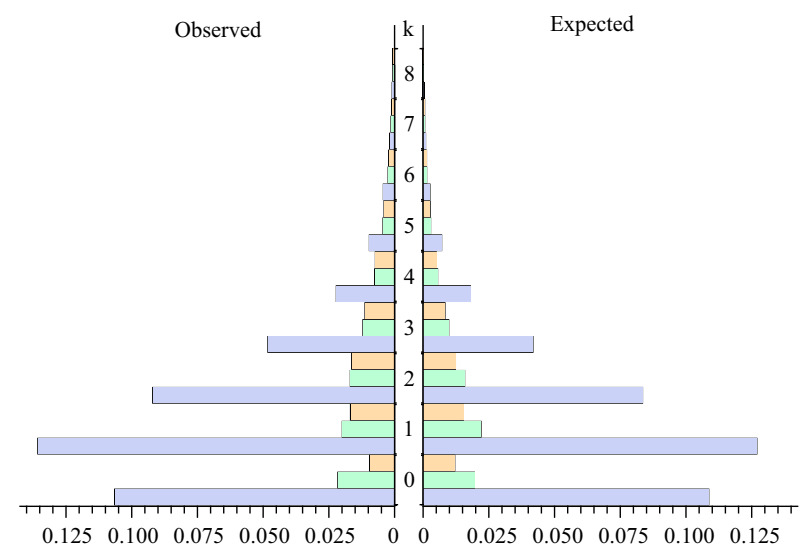

Fig. 3 Expected (under IBA model with parameters fixed at their MLE) and observed mutational configuration. The $X$-axis represents the proportion of blocks with $\mathrm{k}$ mutations ( $\mathrm{Y}$ axis) for different topologies. Blue bars $=(S$. scrofa, (S. verrucosus, S. cebifrons) $)$, green bars $=(S$. verrucosus, $(S$. cebifrons, S. scrofa $))$ and orange $=($ S. cebifrons, $($ S. verrucosus, $S$. scrofa $))$.

$T_{g f}$ below $70 \mathrm{Ky}(\Delta \ln L<-6$; Fig. $4 \mathrm{~b})$, the time of the earliest human arrival in the region (Mijares et al. 2010), which strongly suggest that humans did not play a role in this admixture.

Our second goal was to put the initial divergence between these species in the context of the climate history during the Pleistocene. Our point estimate for $T_{2}$ that is the deeper speciation event in this study (S. scrofa and S. verrucosus/Sus cebifrons split, Fig. 2) is approximately 4My (Fig. 4b) for both block sizes, with lower 95\% CI much older than $>2.5 \mathrm{My}$ (Plio-Pleistocene transition; Fig. 4b). We estimated the split between the Javan warty pig ( $S$. verrucosus) and the Visayan warty pig (S. cebifrons) ( $\left.T_{1}\right)$ to be between 1.3 and $1.1 \mathrm{My}$ (Fig. $4 \mathrm{~b}$ ) These divergence time estimates agree well with previous analyses based on the same molecular clock (Frantz et al. 2013).

\section{Discussion}

In this study, we show that the extent and the directionality of interspecific admixture between Sunda-shelf Sus species (Fig. 1) is more complex than previously assumed (Frantz et al. 2013). First, our likelihood approach allows us to rule out any major influence of humans in this admixture event. Second, our analysis suggests that ISEA Sus species diverged during warmer periods of the late Pliocene and hybridized during the more frequent glacial periods of the mid-Pleistocene. Finally, and perhaps surprisingly, our analyses suggest that admixture occurred mainly from Sus verrucosus into Sus scrofa, so in the opposite direction than that assumed previously. 


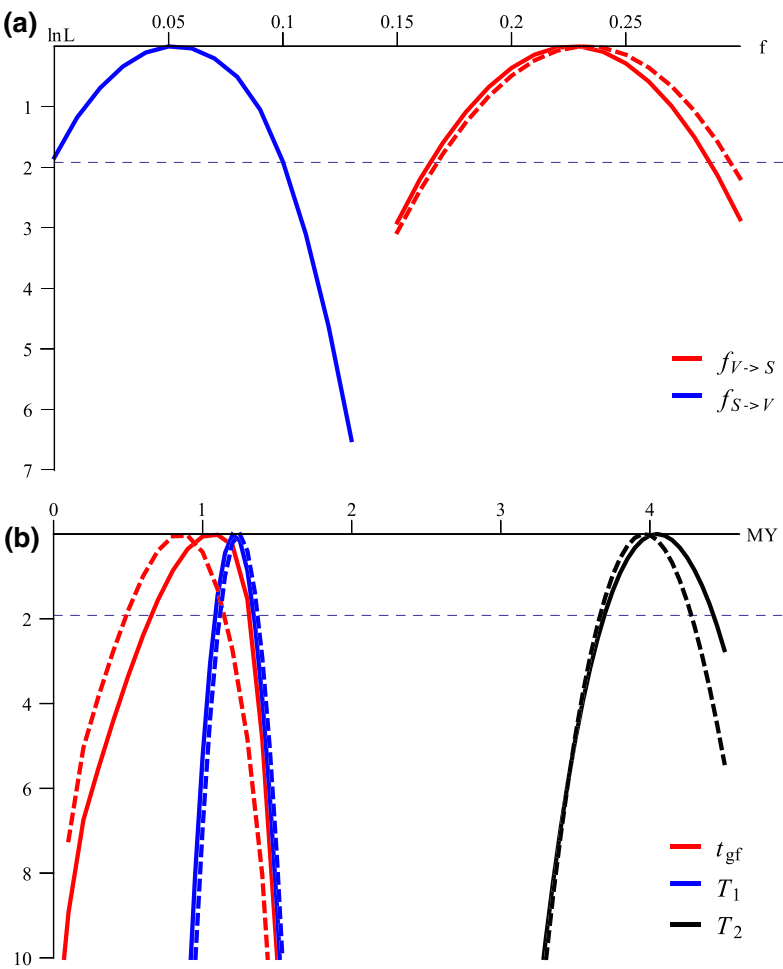

Fig. 4 Marginal support $(\Delta l n L)$ for a) admixture fraction $(f)$ and b) divergence and admixture times. Solid and dashed lines represent $\Delta \ln L$ curves under the IUA_SV and IBA models, respectively. The black dashed horizontal lines delimit the 95\% confidence interval. a) admixture fraction from S. verrucosus into S. scrofa and from S. scrofa into S. verrucosus are shown in red and blue represent respectively b) $T_{2}, T_{1}$ and $T_{g f}$ are shown in black, blue and red respectively.

\section{Fine scale model testing using a likelihood approach}

Models involving interspecific admixture between Sus species on the Sunda-shelf (Fig. 1) fitted our genomic data set significantly better than a simple divergence model without gene flow (Table 1). We estimated a $23 \%$ admixture fraction from the Java Warty pig (S. verrucosus) into the S. scrofa population on Sumatra (Fig. 4). This admixture can explain the large discrepancies found between nuclear and mtDNA phylogenetic analyses that found that S. verrucosus and Sumatran S. scrofa share very similar mtDNA haplotypes and form a monophyletic clade with short external branches (Larson et al. 2005, 2007; Frantz et al. 2013). Moreover, it seems that replacement of mtDNA in either S. verrucosus or S. scrofa from Sumatra was complete as no divergent mtDNA haplotypes have been found by any previous studies (Larson et al. 2005, 2007). This suggests that interspecific admixture can lead to complete (or near-complete) mtDNA replacement even with an admixture fraction of $\sim 23 \%$ and illustrates that phylo- genetic and phylogeographic studies that rely solely on mtDNA can be highly misleading.

Our results also show that previous analyses of these genomes (Frantz et al. 2013), using D-statistics (Green et al. 2010; Durand et al. 2011), only incompletely resolved this interspecific admixture. This is because the D-statistics provide no direct information about the direction of admixture (Green et al. 2010). Frantz et al. 2013 had assumed a history of unidirectional admixture from S. scrofa into S. verrucosus to be able to compute an upper bound for the admixture fraction under this model from ABBA/BABA counts (see Additional File 6 in Frantz et al. 2013). In contrast, the joint distribution of branch lengths, used in our likelihood approach, contains additional information about the direction of admixture and our analysis reveals that S. verrucosus is largely the source of admixture rather than the recipient. While we can show that the IBA model fitted slightly better than the IUA_SV model, the difference was not significant (Table 1). In other words, although our estimate for $f$ (IBA model) from S. scrofa to S. verrucosus was very similar to the fraction estimated by Frantz et al. 2013 ( $4 \%$ vs. $5 \%$ in this study), our $95 \%$ CI for this parameter also include 0 (Fig. 4a). Therefore, while our results unequivocally support that $S$. verrucosus was the most important source of interspecific admixture, we cannot rule out that this species was also a recipient. Together our analyses show that it is important to interpret admixture fractions computed based on D-statistics with caution when the direction of the admixture is unknown.

\section{Natural interspecific admixture on the Sunda-shelf}

Our analysis showed that most of the interspecific admixture between the Sunda-shelf species took place before humans arrived in the region and so anthropogenic disturbances are unlikely to explain this phenomenon. However, while useful, our models are necessarily oversimplistic. For example, we assumed that admixture was a single, instantaneous event. However, the Sunda-shelf was exposed during multiple glacial cycles in the mid-Pleistocene (Voris 2000), which in turn could have lead to many admixture events. Therefore, although we ruled out humans as the cause for most of this admixture, this does not exclude the possibility that a small amount of admixture occurred more recently as a result of anthropogenic disturbances. This is especially true given that previous studies have found that humans most likely translocated Sus species in the region (Groves 1984; Heinsohn 2003; Larson et al. 2005, 2007; Frantz et al. 2013). Thus, the genomic signature left by human-mediated translocation of species 
may be confounded with the signal of large-scale, naturally occurring admixture.

We also evaluated the support of a model of symmetrical admixture (ISA; Table 1). Our model comparison clearly demonstrates that this scenario provides a significantly poorer fit than the IBA model. This is unsurprising given the large difference in admixture fraction under the IBA model $(5 \%$ vs. $23 \%)$. This asymmetry could arise in at least two ways: First, a low effective population size $\left(N_{e}\right)$ of Sumatran S. scrofa - perhaps as a result of a founder event when this species colonized Sumatra from the mainland at the time of admixture could explain this observation. However, the origin of S. scrofa (on the mainland or on ISEA) remains controversial due to the difficulty of inferring demographic events that took place more than 2My ago (Frantz et al. 2013). Alternatively, this discrepancy in the admixture fraction could be the result of greater mate discrimination against hybrids by S. verrucosus. This interpretation is difficult to assess given the very sparse ecological and behavioural data available for the Javanese warty pig S. verrucosus (Blouch 1993). However, such information is available for S. scrofa, an invasive generalist that can easily colonize new environments (Barrios-Garcia \& Ballari 2012). Sus scrofa can be found natively all over Eurasia, in Sumatra and parts of North Africa. Moreover, feral S. scrofa have been able to colonize new ecosystems in Australia, Hawaii, Java, North America and many other parts of the globe in recent years. Thus, given the generalist behaviour of this species, its wide range and its ability to colonize new environments and the relatively narrow range of $S$. verrucosus (restricted to a few areas on Java) mate discrimination against hybrids by the latter would appear more probable. Such an asymmetry in sexual selection against hybrids has been suggested in mice (i.e. Latour et al. 2014). Further research on the ecology and the behaviour of the Java warty pig is needed to better interpret these results, especially given its endangered status. More efficient mate discrimination against hybrids by S. verrucosus would have important consequences for on-going conservation effort. Indeed, one of the major threats to S. verrucosus, listed by the IUCN Wild Pig Specialist Group (Semiadi et al. 2008), is the hybridization with the potentially recently introduced S. scrofa on Java. However, hybrids are difficult to identify in the wild and the extent of this threat remains unknown (Semiadi et al. 2008). Disentangling these two hypotheses (hybrid recruitment vs. founder effect) would provide crucial information for the conservation of S. verrucosus.

Adding parameters to model population size difference between these species did not improve the fit of our models (Table S1, Supporting information). This does of course not imply that these species have the same effective population size, but rather demonstrates that there is little information in the blockwise data to fit more realistic histories (Fig. 3). In contrast, the information contained in linkage across longer stretches of the genome suggests that these species have experienced substantial changes in $N_{e}$. For example, both S. verrucosus and S. cebifrons carry long runs of homozygosity and have a low current $N_{e}$ that was attributed to very recent bottlenecks possibly due to anthropogenic disturbances (Bosse et al. 2012; Frantz et al. 2013). In addition, all three species showed demographic signals consistent with long-term bottlenecks during the Pleistocene (Frantz et al. 2013). Lastly, as discussed above, in the IBA model, $f$ and $N_{e}$ are almost entirely confounded (Lohse et al. 2011), thus it is not surprising that there is no additional power to estimate variation in the latter.

\section{The impact of Plio-Pleistocene glaciations on the evolutionary history of Sus}

Our analyses show that the divergence between Sundashelf Sus species took place during the Pliocene around $4 \mathrm{My}$ ago (Fig. $4 \mathrm{~b}$ ). Moreover, we found that the divergence between S. verrucosus and Sus cebrifrons occurred over $1.2 \mathrm{My}$ ago, during the early Pleistocene before the sharp climatic transition $\sim 700 \mathrm{Ka}$ (Fig. $4 \mathrm{~b}$ ). This suggests that the milder climatic fluctuations of the Pliocene (Fig. 1) allowed for dispersal between 'islands' during short glacial period and subsequent isolation during long warm periods (due to high sea level), while the longer and more frequent ice ages of the late Pleistocene which resulted in longer exposure of the Sundashelf (due to low sea level) led to a partial merging of gene pools. Thus, the Plio-Pleistocene climatic fluctuations may have had the reverse effect in ISEA compared to more temperate regions such as Europe, in which glacial maximas induce divergence (assemblage of refugia) and interglacial periods induce range expansions and hybridization (i.e. Hewitt 2000, 2004, 2011; Schmitt 2007). However, our large confidence intervals around time parameters (divergence and admixture) as well as our model assumptions (single instantaneous admixture) do not provide the necessary resolution to correlate these events with individual glacial cycles during the Plio-Pleistocene era (Zachos et al. 2001; Elderfield et al. 2001). Further studies using larger data sets, increasingly sophisticated methods and combining historical inferences from multiple species will shed light on the mechanisms that generated and erased biodiversity in this mega biodiverse region of the world (Myers et al. 2000). 


\section{Acknowledgements}

This paper is dedicated to the memory of our colleague William Oliver, who devoted his life to conservation biology. We would like to thank members of the IUCN Wild Pig Specialists Group, in particular, Erik Meijaard and Gono Semiadi for their fruitful insights into the conservation status and challenges of S. verrucosus. This project was financially supported by a European Research Council grant (ERC-2009-AdG: 249894) and a junior research fellowship from the Natural Environmental Research Council (NE/I020288/1) to KL.

\section{References}

Barrios-Garcia MN, Ballari SA (2012) Impact of wild boar (Sus scrofa) in its introduced and native range: a review. Biological Invasions, 14, 2283-2300.

Bird MI, Taylor D, Hunt C (2005) Palaeoenvironments of insular Southeast Asia during the Last Glacial Period: a savanna corridor in Sundaland? Quaternary Science Reviews, 24, 2228-2242.

Blouch RA (1993) The Java Warty Pig (Sus verrucosus). In: Pigs, Peccaries and Hippos: Status Survey and Conservation Action Plan (ed. Oliver WLR), pp. 129-136. IUCN, Gland, Switzerland.

Bosse M, Megens H-J, Madsen O et al. (2012) Regions of homozygosity in the porcine genome: consequence of demography and the recombination landscape. PLoS Genetics, 8, e1003100.

Cahill JA, Green RE, Fulton TL et al. (2013) Genomic evidence for island population conversion resolves conflicting theories of polar bear evolution. (ed. Nachman MW). PLoS Genetics, 9, e1003345.

Cannon CH, Morley RJ, Bush ABG (2009) The current refugial rainforests of Sundaland are unrepresentative of their biogeographic past and highly vulnerable to disturbance. Proceedings of the National Academy of Sciences of the United States of America, 106, 11188-11193.

Durand EY, Patterson N, Reich D, Slatkin M (2011) Testing for ancient admixture between closely related populations. Molecular Biology and Evolution, 28, 2239-2252.

Elderfield H, Ferretti P, Greaves M et al. (2012) Evolution of ocean temperature and ice volume through the mid-Pleistocene climate transition. Science (New York, N.Y.), 337, 704-709.

Frantz LA, Schraiber JG, Madsen O et al. (2013) Genome sequencing reveals fine scale diversification and reticulation history during speciation in Sus. Genome Biology, 14, R107.

Gathorne-Hardy FJ, Davies RG, Eggleton P, Jones DT (2002) Quaternary rainforest refugia in south-east Asia: using termites (Isoptera) as indicators. Biological Journal of the Linnean Society, 75, 453-466.

Gongora J, Cuddahee RE, Nascimento FFD et al. (2011) Rethinking the evolution of extant sub-Saharan African suids (Suidae, Artiodactyla). Zoologica Scripta, 40, 327-335.

Green RE, Krause J, Briggs AW et al. (2010) A draft sequence of the Neandertal genome. Science (New York, N.Y.), 328, 710-722.

Groenen MAM, Archibald AL, Uenishi H et al. (2012) Analyses of pig genomes provide insight into porcine demography and evolution. Nature, 491, 393-398.

Gronau I, Hubisz MJ, Gulko B, Danko CG, Siepel A (2011) Bayesian inference of ancient human demography from individual genome sequences. Nature Genetics, 43, 1031-1034.
Groves CP (1984) Of mice and men and pigs in the Indo-Australian Archipelago. Canberra Anthropology, 7, 1-19.

Hall R (1998) The plate tectonics of Cenozoic SE Asia and the distribution of land and sea. In: Biogeography and Geological Evolution of SE Asia (eds Hall R, Holloway JD), pp. 99-131. Backhuys Publishers, Leiden, the Netherlands.

Hearn J, Stone GN, Bunnefeld L et al. (2014) Likelihood-based inference of population history from low-coverage de novo genome assemblies. Molecular Ecology, 23, 198-211.

Heinsohn T (2003) Animal translocation: long-term human influences on the vertebrate zoogeography of Australasia (natural dispersal versus ethnophoresy). Australian Zoologist, 32, 351-376.

Hewitt G (2000) The genetic legacy of the Quaternary ice ages. Nature, 405, 907-913.

Hewitt GM (2004) Genetic consequences of climatic oscillations in the Quaternary. Philosophical Transactions of the Royal Society of London. Series B, Biological Sciences, 359, 183-195; discussion 195.

Hewitt GM (2011) Quaternary phylogeography: the roots of hybrid zones. Genetica, 139, 617-638.

Larson G, Dobney K, Albarella U et al. (2005) Worldwide phylogeography of wild boar reveals multiple centers of pig domestication. Science (New York, N.Y.), 307, 1618-1621.

Larson G, Cucchi T, Fujita M et al. (2007) Phylogeny and ancient DNA of Sus provides insights into neolithic expansion in Island Southeast Asia and Oceania. Proceedings of the National Academy of Sciences of the United States of America, 104, 4834-4839.

Latour Y, Perriat-sanguinet M, Caminade P et al. (2014) Sexual selection against natural hybrids may contribute to reinforcement in a house mouse hybrid zone. Proceedings. Biological sciences/The Royal Society, 281, 20132733.

Lawniczak MKN, Emrich SJ, Holloway AK et al. (2010) Widespread divergence between incipient Anopheles gambiae species revealed by whole genome sequences. Science (New York, N.Y.), 330, 512-514.

Li H, Handsaker B, Wysoker A et al. (2009) The sequence alignment/map format and SAMtools. Bioinformatics (Oxford, England), 25, 2078-2079.

Lohse K, Frantz LAF (2014) Neandertal admixture in Eurasia confirmed by maximum likelihood analysis of three genomes. Genetics, 196, 1241-1251.

Lohse K, Harrison RJ, Barton NH (2011) A general method for calculating likelihoods under the coalescent process. Genetics, 189, 977-987.

Meijaard E, d'Huart JP, Oliver WLR (2011) Family Suidae (Pigs). In: Handbook of the Mammals of the World. Volume 2 (eds Wilson DE, Mittermeier RA), pp. 248-291. Lynx Edicions, Barcelona, Spain.

Mijares AS, Détroit F, Piper P et al. (2010) New evidence for a 67,000-year-old human presence at Callao Cave, Luzon, Philippines. Journal of Human Evolution, 59, 123-132.

Miller KG, Kominz MA, Browning JV et al. (2005) The Phanerozoic record of global sea-level change. Science (New York, N.Y.), 310, 1293-1298.

Myers N, Mittermeier RA, Mittermeier CG, Fonseca GAB, Kent J (2000) Biodiversity hotspots for conservation priorities. Nature, 403, 853-858.

Oliver W (2008). Sus cebifrons. In: IUCN 2014. IUCN Red List of Threatened Species. Version 2014.1. <www.iucnredlist. org >. Downloaded on 22 May 2014. 
Paudel Y, Madsen O, Megens H-J et al. (2013) Evolutionary dynamics of copy number variation in pig genomes in the context of adaptation and domestication. BMC Genomics, 14, 449.

Rohland N, Reich D, Mallick S et al. (2010) Genomic DNA sequences from mastodon and woolly mammoth reveal deep speciation of forest and savanna elephants. PLoS Biology, 8, e1000564.

Schmitt T (2007) Molecular biogeography of Europe: pleistocene cycles and postglacial trends. Frontiers in Zoology, 4, 11.

Semiadi G, Meijaard E, Oliver W (2008). Sus verrucosus. In: IUCN 2013. IUCN Red List of Threatened Species. Version 2014.1. <www.iucnredlist.org>. Downloaded on 22 May 2014.

Slik JWF, Aiba S-I, Bastian M et al. (2011) Soils on exposed Sunda shelf shaped biogeographic patterns in the equatorial forests of Southeast Asia. Proceedings of the National Academy of Sciences of the United States of America, 108, 12343-12347.

Stewart JR, Lister AM, Barnes I, Dalén L (2010) Refugia revisited: individualistic responses of species in space and time. Proceedings. Biological sciences/The Royal Society, 277, 661-671.

Tortereau F, Servin B, Frantz L et al. (2012) A high density recombination map of the pig reveals a correlation between sex-specific recombination and GC content. BMC Genomics, $13,586$.

Voris HK (2000) Maps of Pleistocene sea levels in Southeast Asia: shorelines, river systems and time durations. Journal of Biogeography, 27, 1153-1167.

Wurster CM, Bird MI, Bull ID et al. (2010) Forest contraction in north equatorial Southeast Asia during the Last Glacial Period. Proceedings of the National Academy of Sciences of the United States of America, 107, 15508-15511.
Zachos J, Pagani M, Sloan L, Thomas E, Billups K (2001) Trends, rhythms, and aberrations in global climate 65 Ma to present. Science (New York, N.Y.), 292, 686-693.

L.A.F.F., K.L. and M.A.M.G. designed the study. L.A.F.F., O.M. and H.-J.M. carried out sequence alignment and SNP calling. K.L. and L.A.F.F. analysed the data. L.A.F.F. and K.L. wrote the manuscript with input from O.M., H.-J.M. and M.A.M.G.

\section{Data accessibility}

BAM files: EBI Sequence Read Archive ERP001813.

Mathematica notebook and input files (mutational configurations for 500-bp and 1-kbp blocks): Dryad doi:10.5061/dryad.1q0h6.

\section{Supporting information}

Additional supporting information may be found in the online version of this article.

Table S1. Support for model with extra $N_{e}$ parameters.

Data S1. Supporting Mathematica notebook. 